\title{
A case of Naegleria fowleri related primary amoebic meningoencephalitis in China diagnosed by next-generation sequencing

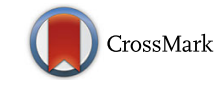

Qiang Wang ${ }^{1,2+}$, Jianming $\mathrm{Li}^{1+}$, Jingkai $\mathrm{Ji}^{3,6+}$, Liuqing Yang ${ }^{1}$, Li Chen ${ }^{1}$, Rongrong Zhou ${ }^{1}$, Yang Yang ${ }^{1,2}$,
Haixia Zheng ${ }^{1}$, Jing Yuan ${ }^{1}$, Liqiang $\mathrm{Li}^{3,6}$, Yuhai $\mathrm{Bi}^{1,2}$, George F. Gao ${ }^{1,2,4,5}$, Jinmin Ma ${ }^{3,6,7^{*}}$ and Yingxia Liu

\begin{abstract}
Background: Primary amoebic meningoencephalitis (PAM), caused by Naegleria fowleri, is a rare protozoan infectious disease in China. A fatality rate of over $95 \%$ had been reported due to extremely rapid disease progression in the USA and other countries. Rapid and precise identification of the causative agent is very important to clinicians for guiding their choices for administering countermeasures in the clinic. In this report, we applied the next-generation sequencing (NGS) method to rapidly show that $N$. fowleri was the causative agent of a fatal case involving a 42-year-old man with severe PAM disease, the first reported in mainland China.

Case presentation: A 42-year old male in a deep coma was admitted to Shenzhen Third People's Hospital, a special medical care unit with expertise in infectious diseases. Increased intracranial pressure was detected. The cerebrospinal fluid (CSF) sample was found to be red and cloudy with increased leukocyte and protein levels. While bacterial cultures with CSF were negative, $N$. fowleri was determined to be the causative agent with NGS.

Amphotericin B (AmB), a drug with anti-amoeba activity, was used immediately, but the treatment came too late and the patient died 2 days after the NGS confirmation.

Conclusion: In this paper, we reported a case of PAM disease for the first time in mainland China. NGS was used for rapid diagnosis and provided guidance for prescribing medications. However, the patient died due to a late admission amid advanced PAM disease. Early detection of N. fowleri is necessary in order to select effective drug treatments and control the disease progression. Despite the negative survival outcome, NGS was shown to be a promising method of rapid and precise identification of $\mathrm{N}$. fowleri.
\end{abstract}

Keywords: Naegleria fowleri, Primary amoebic meningoencephalitis, Amoeba, Next-generation sequencing

\section{Background}

Primary amoebic meningoencephalitis (PAM) is an acute and rapidly fatal disease of the central nervous system, caused by infection with Naegleria fowleri, a thermophilic free-living amoeba found in warm freshwater such as lakes, ponds, rivers and hot springs [1]. Contaminated tap water could also be a source of infection [2]. N. fowleri usually infects people through the nose cavity when

\footnotetext{
* Correspondence: majinmin@genomics.cn; yingxialiu@hotmail.com ${ }^{+}$Qiang Wang, Jianming Li and Jingkai Ji contributed equally to this work. ${ }^{3}$ BGI-Shenzhen, Shenzhen 518083, China

${ }^{1}$ Shenzhen Key Laboratory of Pathogen and Immunity, State Key Discipline of Infectious Disease, Shenzhen Third People's Hospital, 29 Bulan Rd, Shenzhen 518112, China

Full list of author information is available at the end of the article
}

in contact with contaminated water. N. fowleri is commonly known as a "brain-eating amoeba" because of its induction of severe encephalitis in the brain upon infection, with a fatality rate of over $95 \%$ [3]. There are over 40 species of Naegleria, but only N. fowleri infections results in PAM [1]. PAM has a rapid, acute disease progression, with an incubation period varying from 2 to 15 days, and death typically occurring 3-7 days after the onset of symptoms [1]. N. fowleri has been reported worldwide including America, Australia, Thailand, Hong Kong and Taiwan [4], and about 300 cases in total have been reported in 50 years since the first case was reported in 1965 [3]. Prompt diagnosis is important in order to start treatment as soon as possible. However,

(c) The Author(s). 2018 Open Access This article is distributed under the terms of the Creative Commons Attribution 4.0 International License (http://creativecommons.org/licenses/by/4.0/), which permits unrestricted use, distribution, and reproduction in any medium, provided you give appropriate credit to the original author(s) and the source, provide a link to the Creative Commons license, and indicate if changes were made. The Creative Commons Public Domain Dedication waiver (http://creativecommons.org/publicdomain/zero/1.0/) applies to the data made available in this article, unless otherwise stated. 
prompt diagnosis is not easy for those who had no experience in treatment of $N$. fowleri infection and PAM is often misdiagnosed because no distinctive differences in diagnosis exist to distinguish PAM from bacterial meningoencephalitis [5]. In this study, we report using the next generation sequencing (NGS) method for prompt diagnosis of a $N$. fowleri-related infection, the first case in mainland China.

\section{Case presentation}

On 20th August 2016, a 42-year-old man with a fever $\left(38.4{ }^{\circ} \mathrm{C}\right)$ was admitted to a local hospital in Hangzhou City, China. He lived in Shenzhen City, but was visiting Hangzhou at the time. His disease began with a headache 1 day earlier. Upon admission, blood tests showed a leukocytosis of $10.48 \times 10^{9} / \mathrm{L}$ ( $80 \%$ neutrophils). On the night of admission, a lumbar puncture showed high levels of white blood cells (WBCs, 1170 cells/ $\mu \mathrm{L}, 83 \%$ neutrophils), a protein concentration of $3.0 \mathrm{~g} / \mathrm{L}$ and a glucose concentration of $1.0 \mathrm{mmol} / \mathrm{L}$ in the cerebrospinal fluid (CSF) sample. He was treated with $2.0 \mathrm{~g}$ of the antimicrobial ceftriaxone and his situation was not improved.

One day later, the patient's speech became incoherent and he experienced dyspnea. He was transferred to the intensive care unit and endotracheal intubation was applied. He was administered combined therapy comprising of antibiotics (meropenem and linezolid) and an anti-inflammatory drug (dexamethasone). During the night, the patient was found in a coma with dilated bilateral pupils. Computed tomography scans of his brain illustrated hydrocephalus, cerebral edema and foggy brain ventricles. Ventricle puncture for external CSF drainage was performed to reduce intracranial pressure. Four days after admission, microbial culture results of the CSF samples were negative for bacteria and fungi.

On 31th August, the patient was taken back to Shenzhen at the request of his family, and transferred to Shenzhen Third People's Hospital, the key institute for handling infectious disease cases. At the time of admission, he was already in a deep coma and unresponsive to simple cues. A blood test showed a leukocytosis of $14.72 \times 10^{9} / \mathrm{L}$ (93.3\% neutrophils). The CSF appeared light red in color and was mixed with cloudy debris. The WBC count in the CSF had increased substantially to 52,860 cells/ $\mu \mathrm{L}$ (79\% lymphocytes), whereas the protein level had increased to $49.96 \mathrm{~g} / \mathrm{L}$ and the glucose concentration was $0.11 \mathrm{mmol} / \mathrm{L}$. Computed tomography scans showed severe brain swelling. The shape of the cerebral ventricles had become twisted and almost disappeared (Fig. 1).

His CSF sample was immediately sent for pathogen detection by NGS at BGI-Shenzhen. Briefly, the CSF sample was centrifuged at $10,000 \times \mathrm{g}$ for $10 \mathrm{~min}$ and the

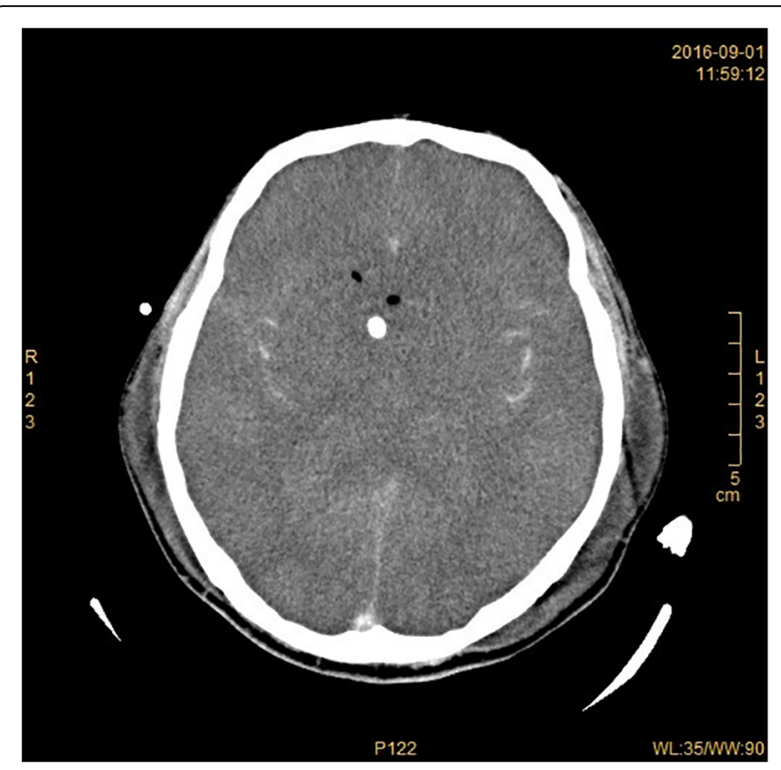

Fig. 1 Computed tomography scan of the patient brain with primary amoebic meningoencephalitis

precipitate was used for nucleic acid extraction using the QIAamp DNA Mini Kit (Qiagen), following manufacturer instructions. NGS was performed on the newly developed BGISEQ-500 platform [6]. The sequencing detection identified 65,658 (out of $246,938,950$ ) sequence reads $(0.0266 \%)$ uniquely corresponding to the $N$. fowleri genome (Fig. 2b) [7], and these reads covered a high percentage of the genome (Fig. 2a). When the reads from the human host were excluded, $N$. fowleri reads were comprised the most of any microbial species, accounting for $0.3689 \%$ of total microbial reads and unknown or unclassified reads (Fig. 2c), and more than the total reads of bacterium or fungi (Additional file 1). A 1.6-kb consensus sequence of the $18 \mathrm{~S}$ ribosomal RNA of $N$. fowleri was assembled (GenBank accession no.KY062165) and found to be $99.99 \%$ identical to a reference $N$. fowleri sequence (GenBank accession no. U80059). N. fowleri infection was confirmed by PCR (Fig. 3) using the specific primers NFITS-FW (TGAAAACCTTTTTTCCATTTA CA) and NFITS-RV (AATAAAAGATTGACCATTTGA AA) covering the $5.8 \mathrm{~S}$ ribosomal DNA and internal transcribed spacer 2 [4]. Therefore, all these results indicate that the patient was infected with $N$. fowleri. In addition, patient history collected from family members showed that the man was splashed by lake water when he attended the Water-Splashing Festival in Shenzhen approximately 1 weeks before symptoms onset, which may be the source of infection. Anti-amoebic drugs including amphotericin B (AmB) at $50 \mathrm{mg} /$ day and fluconazole $0.4 \mathrm{~g} /$ day were immediately administered. Unfortunately, symptoms did not improve and the patient's family requested to cease medical treatment 2 days later. Life 

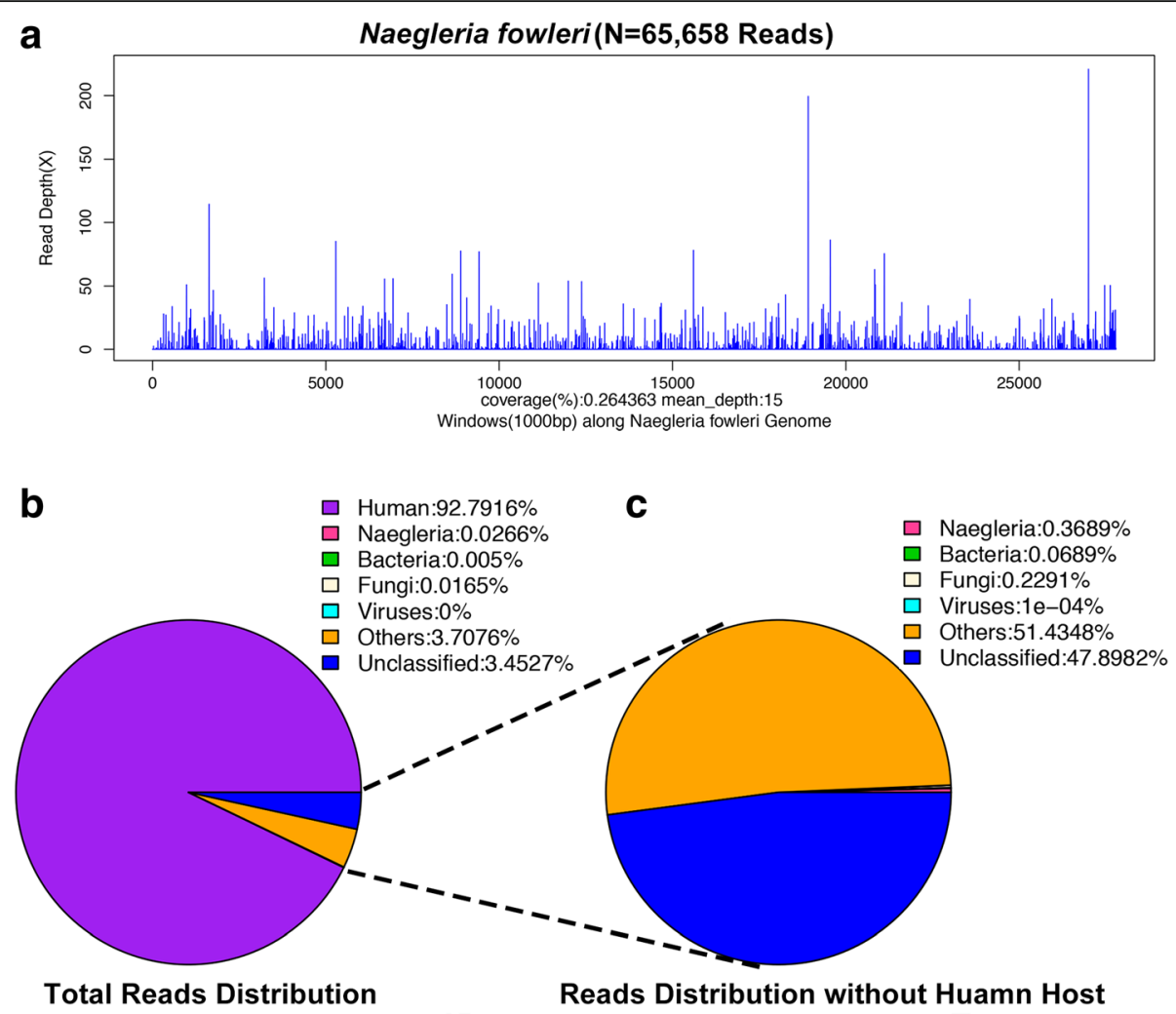

Fig. 2 Diagnosis of $N$. fowleri infection using the NGS method. a. mapping of $N$. fowleri reads on the genome. b. Reads distribution of total DNA in the CSF sample. c. Reads distribution of microbes and unknown reads in the absence of human host reads

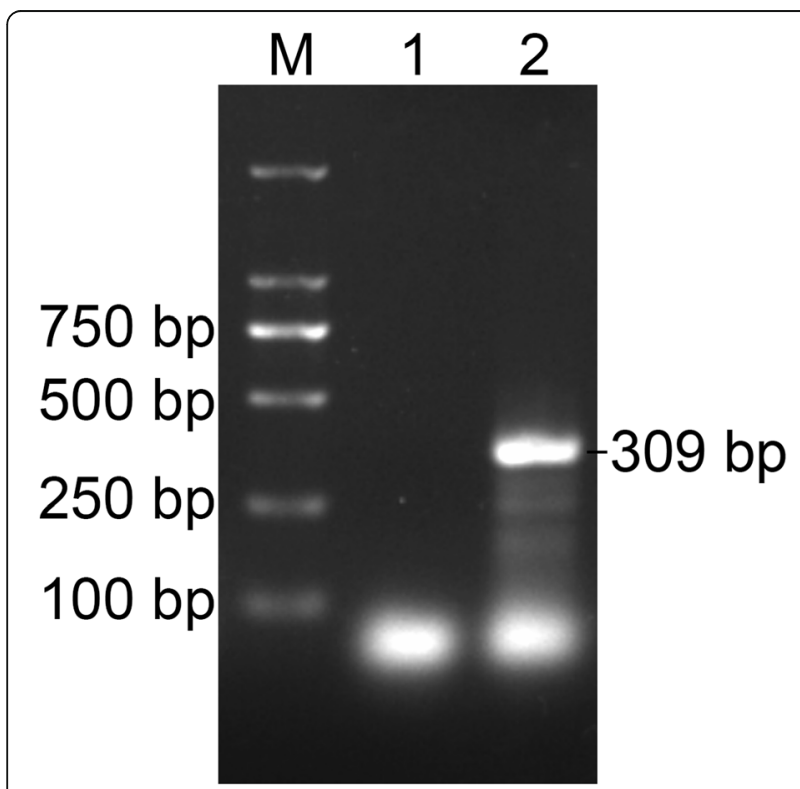

Fig. 3 PCR detection of $N$. fowleri from CSF specimen. Lane M: DNA ladder. Lane 1: Negative control. Lane 2: 309-bp PCR product of the $5.8 \mathrm{~S}$ ribosomal DNA and internal transcribed spacer 2 region of N. fowleri support was withdrawn and the patient was pronounced dead on 3rd September.

\section{Discussion}

We report here a fatal case of PAM caused by $N$. fowleri infection, in which infection likely occurred after the patient had direct contact with lake water 2 weeks before the onset of symptoms. This is the first case of $N$. fowler$i$-related PAM reported in mainland China. Only two cases were report in Hong Kong and Taiwan, respectively. Although three suspected fatal PAM cases in mainland China had been documented in Chinese journals during 1978, 1990 and 2001, respectively (Table 1), the causative pathogen was never conclusively identified. Increased levels of leukocytes and protein, and a decrease in glucose levels, were consistently observed in all six cases during the disease course.

PAM is acute and aggressive, with death typically occurring 3-7 days after onset of symptoms. Early diagnosis and rapid treatment with the appropriate drugs are key factors for effective therapy. In the current case, the man had a suspected incubation period of 5 days and died 15 days after disease onset. Although $N$. fowleri was identified in only 2 days after his admission to Shenzhen Third People's Hospital, the patient was already in a 
Table 1 Primary amoebic meningoencephalitis cases reported in China and neighboring places

\begin{tabular}{|c|c|c|c|c|c|c|c|c|c|c|c|}
\hline \multirow[t]{2}{*}{ Age } & \multirow[t]{2}{*}{ Gender } & \multirow[t]{2}{*}{ Location } & \multirow[t]{2}{*}{ Year } & \multicolumn{5}{|c|}{ First CSF after admission } & \multirow{2}{*}{$\begin{array}{l}\text { Amoebicidal } \\
\text { Treatment }\end{array}$} & \multirow{2}{*}{$\begin{array}{l}\text { Days from } \\
\text { symptom } \\
\text { onset to death }\end{array}$} & \multirow{2}{*}{ Diagnosis } \\
\hline & & & & $\begin{array}{l}\text { WBC } \\
\text { (cells/ } / \mu \mathrm{L})\end{array}$ & $\begin{array}{l}N \\
\%\end{array}$ & $\begin{array}{l}L \\
\end{array}$ & $\begin{array}{l}\text { Glucose } \\
\text { (mmol/L) }\end{array}$ & $\begin{array}{l}\text { Protein }(\mathrm{g} / \\
\mathrm{L})\end{array}$ & & & \\
\hline$\overline{17}$ & male & Henan $^{b}$ & 1978 & 50 & 5 & 95 & 2.95 & 1.52 & NA & 10 days & $\begin{array}{l}\text { Amoebic trophozoites were } \\
\text { observed in the brain tissue } \\
\text { after death. }\end{array}$ \\
\hline 21 & male & Beijing $^{b}$ & 1990 & 300 & 70 & 30 & 3.4 & 0.5 & NA & 7 days & $\begin{array}{l}\text { Amoebic trophozoites were } \\
\text { found in the CSF after death. }\end{array}$ \\
\hline 23 & male & Hainan $^{b}$ & 2001 & 22,000 & 85 & NA & 2.2 & 2 & none & 6 days & $\begin{array}{l}\text { Amoebic trophozoites were } \\
\text { detected in the CSF before death. }\end{array}$ \\
\hline 38 & male & $\begin{array}{l}\text { Hong } \\
\text { Kong }\end{array}$ & 1993 & NA & NA & NA & NA & NA & AmB, rifampicin & survival & $\begin{array}{l}\text { Amoeba were observed in an } \\
\text { abscess biopsy. }\end{array}$ \\
\hline 75 & male & Taiwan & 2011 & 560 & 90.5 & 5 & 2.2 & 9.5 & $A m B$ & 25 days $^{a}$ & $\begin{array}{l}\text { Amoebic trophozoites were } \\
\text { detected in the CSF. }\end{array}$ \\
\hline 42 & male & Shenzhen & 2016 & 1170 & 83 & 12 & 1 & 3 & $\begin{array}{l}\text { AmB, } \\
\text { fluconazole }\end{array}$ & 15 days & Next-generation sequencing \\
\hline
\end{tabular}

CSF Cerebrospinal fluid, WBC White blood cells, $N$ Neutrophils, $L$ Lymphocytes, NA Not available

${ }^{\mathrm{a}}$ Days after the start of $\mathrm{AmB}$ treatment

${ }^{\mathrm{b}}$ Suspected cases documented in Chinese journals without an English citation version

comatose state, indicating advanced, severe illness. Anti-amoeba medical treatment was initiated 11 days after the onset of disease symptoms; therefore, treatment with the appropriate drugs likely occurred too late to abate the acute phase of PAM.

NGS played a critical role in the accurate diagnosis of $N$. fowleri in the present case. By contrast, the amoeba was not identified in culture. Therefore, NGS constitutes a rapid and accurate method for the identification of pathogens and is particularly helpful in the diagnosis of diseases with unknown causes.

Although no clinically approved specific drugs were available, AmB and miltefosine were reported in successful treatment of $N$. fowleri related PAM. AmB, which is a common drug in clinic and is primarily used as an antifungal medication, has an amoebicidal effect and has been used to treat PAM since 1970. However, the recovery rate with AmB is only 5\% (15 recoveries/300 cases worldwide), and this drug can be toxic to humans [3]. Miltefosine, a drug developed against breast cancer and Leishmania infections, possesses activity against free-living amoeba species in vitro $[8,9]$, and is now recommended for the treatment of PAM by the Centers for Disease Control and Prevention in the USA [10], while is not currently available in China. In the United States, 143 PAM cases have been reported, with only four survivors between 1962 and $2016[11,12]$. Notably, two of the four survivors were related to miltefosine treatment. In 2013 an American girl was diagnosed as PAM about $36 \mathrm{~h}$ after symptom onset and was given the recommended miltefosine therapy. She recovered completely without any neurologic impairment [13]. In contrast, an 8 years-old boy was identified with PAM more than 3 days after disease onset. He survived with brain damage although he was also given the recommended miltefosine therapy [14]. Although miltefosine-containing treatment regimen could offer a survival advantage for patients with fatal infections by free-living amoeba, recovery is not assured, since at least three fatal PAM cases were also reported with receiving miltefosine treatment [14, 15]. In addition, miltefosine should be administered in combination with $\mathrm{AmB}$ and other drugs, such as rifampin and fluconazole, as soon as possible after diagnosing PAM. In addition to drug treatment, physical procedures like CSF drainage, hyperosmolar therapy, moderate hyperventilation and hypothermia have been employed in the treatment of PAM $[3,16]$.

Although PAM is a rare disease in China, the rapid disease process and very high fatality rate make it a very dangerous disease. Unfortunately, we have no data about the distribution range of the pathogenic $N$. fowleri in mainland China. More related research should be carried out to analyze the epidemiological risk of $N$. fowleri in China. On the other hand, development of more specific drugs against $N$. fowleri should be put on schedule.

Most previous cases of PAM have been found to have been infected with $N$. fowleri during recreational activities involving freshwater [17]. In the current case, the patient participated in a Water-Splashing Festival several days before the onset of disease; it is therefore likely that contaminated water was the source of infection. This case is a warning that care should be taken when participating in recreational activities involving freshwater due to the possibility of contamination.

\section{Conclusion}

In the current report, a $N$. fowleri-related PAM was rapidly diagnosed using NGS. Although the patient died, 
the NGS method was shown to be of use to rapidly identify the causative pathogen in the clinic, especially for PAM. The NGS method should be more widely applied in clinical practices, helping physicians on diagnosis and advising on choice of medical countermeasures in the clinic to save lives.

\section{Additional file}

Additional file 1: Microbe reads counts. Other microbe reads including that of bacterium, fungi and virus were listed in the excel table. (XLSX $19 \mathrm{~kb}$ )

\section{Abbreviations}

AmB: Amphotericin B; CSF: Cerebrospinal fluid; N. fowleri: Naegleria fowleri; NGS: Next-generation sequencing; PAM: Primary amoebic meningoencephalitis

\section{Funding}

This work was supported by the Shenzhen Science and Technology Research and Development Project (JCYJ20160427151920801 and JCYJ20160427153524391), the Shenzhen Healthcare Research Project (201601029), the National Science and Technology Major Project (2016ZX10004222). G.F.G. is a leading principal investigator of the NSFC Innovative Research Group (81621091). Y.B. is supported by the Youth Innovation Promotion Association of Chinese Academy of Sciences (CAS) (2017122).

The funders did not play a role in the design of the study and collection, analysis, and interpretation of data and in writing the manuscript.

\section{Availability of data and materials}

The N. fowleri18s ribosomal RNA sequence assembled using the NGS data was submitted to GenBank (accession no.KY062165). Other microbe reads including that of bacterium, fungi and virus were listed in Additional file 1 (Excel table).

\section{Authors' contributions}

$\mathrm{QW}$, $J \mathrm{~L}$ and $J J$ contribute equally to this paper. QW wrote the manuscript. QW, LY, LC, RZ, YY and HZ collected and treated the CSF samples. JL and JY were the physicians who treated the patient and provided professional comments to the manuscript. QW, YB, GG, JM and YL analyzed the clinical data. JJ, LL and JM analyzed the NGS data. All authors have read and approved the final manuscript.

\section{Ethics approval and consent to participate}

Not applicable.

\section{Consent for publication}

Written informed consent was obtained from the patient's family member for publication of this case report. A copy of the written consent is available for review by the Editor of this journal.

\section{Competing interests}

The authors declare that they have no competing interests.

\section{Publisher's Note}

Springer Nature remains neutral with regard to jurisdictional claims in published maps and institutional affiliations.

\section{Author details}

'Shenzhen Key Laboratory of Pathogen and Immunity, State Key Discipline of Infectious Disease, Shenzhen Third People's Hospital, 29 Bulan Rd, Shenzhen 518112, China. ${ }^{2}$ CAS Key Laboratory of Pathogenic Microbiology and Immunology, Collaborative Innovation Center for Diagnosis and Treatment of Infectious Disease, Institute of Microbiology, Center for Influenza Research and Early-warning (CASCIRE), Chinese Academy of Sciences, Beijing 100101, China. ${ }^{3}$ BGI-Shenzhen, Shenzhen 518083, China. ${ }^{4}$ Office of Director-General, Chinese Center for Disease Control and
Prevention, Beijing 102206, China. ${ }^{5}$ Savaid Medical School, University of Chinese Academy of Sciences, Beijing 100049, China. ${ }^{6}$ China National GeneBank, BGI-Shenzhen, Shenzhen 518120, China. 'Laboratory of Genomics and Molecular Biomedicine, Department of Biology, University of

Copenhagen, Copenhagen, Denmark.

Received: 9 November 2017 Accepted: 19 July 2018

Published online: 28 July 2018

\section{References}

1. Yoder JS, Eddy BA, Visvesvara GS, Capewell L, Beach MJ. The epidemiology of primary amoebic meningoencephalitis in the USA, 1962-2008. Epidemiol Infect. 2010;138(7):968-75.

2. Yoder JS, Straif-Bourgeois S, Roy SL, Moore TA, Visvesvara GS, Ratard RC, et al. Primary amebic meningoencephalitis deaths associated with sinus irrigation using contaminated tap water. Clin Infect Dis. 2012;55(9):e79-85.

3. Martínez-Castillo M, Cárdenas-Zúñiga R, Coronado-Velázquez D, Debnath A, Serrano-Luna J, Shibayama M. Naegleria fowleri after 50 years: is it a neglected pathogen? J Med Microbiol. 2016;65(9):885-96.

4. De Jonckheere JF. Origin and evolution of the worldwide distributed pathogenic amoeboflagellate Naegleria fowleri. Infect Genet Evol. 2011;11(7): 1520-8.

5. Rocha-Azevedo B, Tanowitz HB, Marciano-Cabral F. Diagnosis of infections caused by pathogenic free-living amoebae. Interdiscip Perspect Infect Dis. 2009;2009:251406

6. Fang $\mathrm{C}$, Zhong $\mathrm{H}$, Lin $\mathrm{Y}$, Chen $\mathrm{B}$, Han $\mathrm{M}$, Ren $\mathrm{H}$, et al. Assessment of the CPAS-based BGISEQ-500 platform for metagenomic sequencing. GigaScience. 2018;7(3) https://doi.org/10.1093/gigascience/gix133.

7. Zysset-Burri DC, Muller N, Beuret C, Heller M, Schurch N, Gottstein B, et al. Genome-wide identification of pathogenicity factors of the free-living amoeba Naegleria fowleri. BMC Genomics. 2014;15:496.

8. Schuster FL, Guglielmo BJ, Visvesvara GS. In-vitro activity of miltefosine and voriconazole on clinical isolates of free-living amebas: Balamuthia mandrillaris, Acanthamoeba spp., and Naegleria fowleri. J Eukaryot Microbiol. 2006:53(2):121-6.

9. Aichelburg AC, Walochnik J, Assadian O, Prosch H, Steuer A, Perneczky G, et al. Successful treatment of disseminated Acanthamoeba sp. infection with miltefosine. Emerg Infect Dis. 2008;14(11):1743-6.

10. CDC-USA. Investigational drug available directly from $C D C$ for the treatment of infections with free-living amebae. 2013. https://www.cdc. $\mathrm{gov} / \mathrm{mmwr} /$ preview $/ \mathrm{mmwrhtml} / \mathrm{mm} 6233 \mathrm{a} 4 . \mathrm{htm}$ ?s_cid=mm6233a4_e. Accessed 8 May 2018.

11. CDC-USA. Naegleria fowleri - case report data \& graphs. 2016. https://www. cdc.gov/parasites/naegleria/graphs.html. Accessed 8 May 2018.

12. Matanock A, Mehal JM, Liu L, Blau DM, Cope JR. Estimation of undiagnosed Naegleria fowleri primary amebic meningoencephalitis, United States. Emerg Infect Dis. 2018;24(1):162-4.

13. Linam WM, Ahmed M, Cope JR, Chu C, Visvesvara GS, da Silva AJ, et al. Successful treatment of an adolescent with Naegleria fowleri primary amebic meningoencephalitis. Pediatrics. 2015;135(3):e744-8.

14. Cope JR, Conrad DA, Cohen N, Cotilla M, DaSilva A, Jackson J, et al. Use of the novel therapeutic agent miltefosine for the treatment of primary amebic meningoencephalitis: report of 1 fatal and 1 surviving case. Clin Infect Dis. 2016;62(6):774-6.

15. Stowe RC, Pehlivan D, Friederich KE, Lopez MA, DiCarlo SM, Boerwinkle VL. Primary amebic meningoencephalitis in children: a report of two fatal cases and review of the literature. Pediatr Neurol. 2017:70:75-9.

16. Heggie TW, Küpper T. Surviving Naegleria fowleri infections: a successful case report and novel therapeutic approach. Travel Med Infect Dis. 2017;16: 49-51.

17. Capewell LG, Harris AM, Yoder JS, Cope JR, Eddy BA, Roy SL, et al. Diagnosis, clinical course, and treatment of primary amoebic meningoencephalitis in the United States, 1937-2013. J Pediatric Infect Dis Soc. 2015;4(4):e68-75. 